\title{
Visualizing the Electrochemical Lithiation/Delithiation Behaviors of Black Phosphorus by In Situ Transmission Electron
}

\section{Microscopy}

Weiwei Xia, ${ }^{\dagger \#}$ Qiubo Zhang, ${ }^{\dagger \#}$ Feng Xu, ${ }^{* \dagger+}$ Hongyu Ma, ${ }^{\S}$ Jing Chen, " Khan Qasim, ॥

Binghui Ge, ${ }^{\perp}$ Chongyang Zhu, ${ }^{\dagger}$ Litao Sun ${ }^{\dagger+}$

$\dagger$ SEU-FEI Nano-Pico Center, Key Laboratory of MEMS of Ministry of Education, Southeast University, Nanjing 210096, China.

* Center for Advanced Materials and Manufacture, Joint Research Institute of Southeast University and Monash University, Suzhou 215123, China

${ }^{\S}$ Research Center for Internet of Things, China University of Mining and Technology, Xuzhou 221008, China

॥ School of Electronic Science and Engineering, Southeast University, Nanjing 210096, China

${ }^{\perp}$ Institute of Physics, Chinese Academy of Sciences, Beijing 100190, China

\footnotetext{
* To whom correspondence should be addressed. Phone: +86-25-83792632. Fax: +86-25-83792939. E-mail: fxu@ seu.edu.cn (F. $\mathrm{Xu})$;

\# These authors contributed equally to this work.
} 

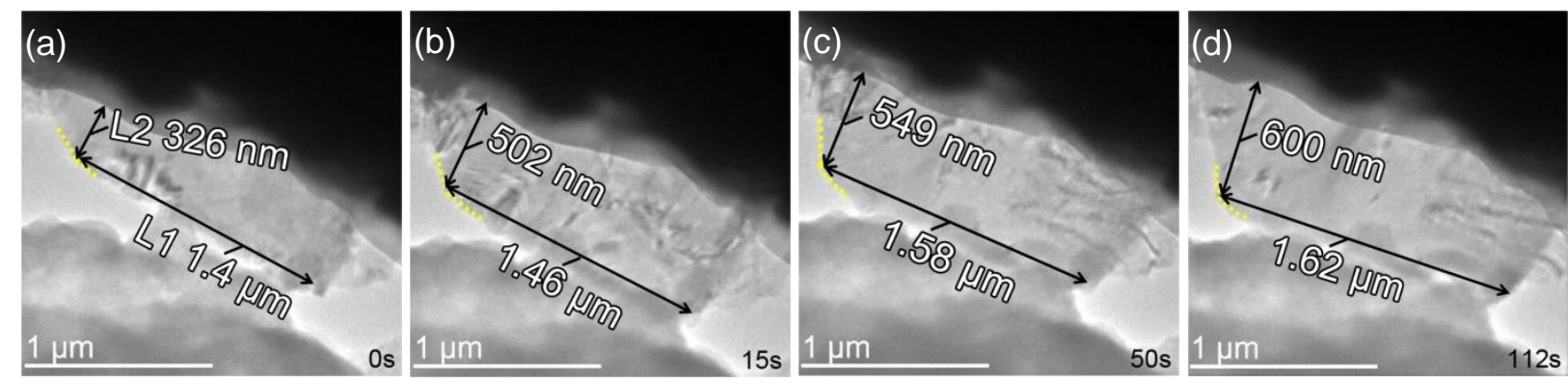

Figure S1. The TEM images of the time-lapse size evolution of BP anode along different directions during first lithiation process, showing obvious anisotropic size expansion. 

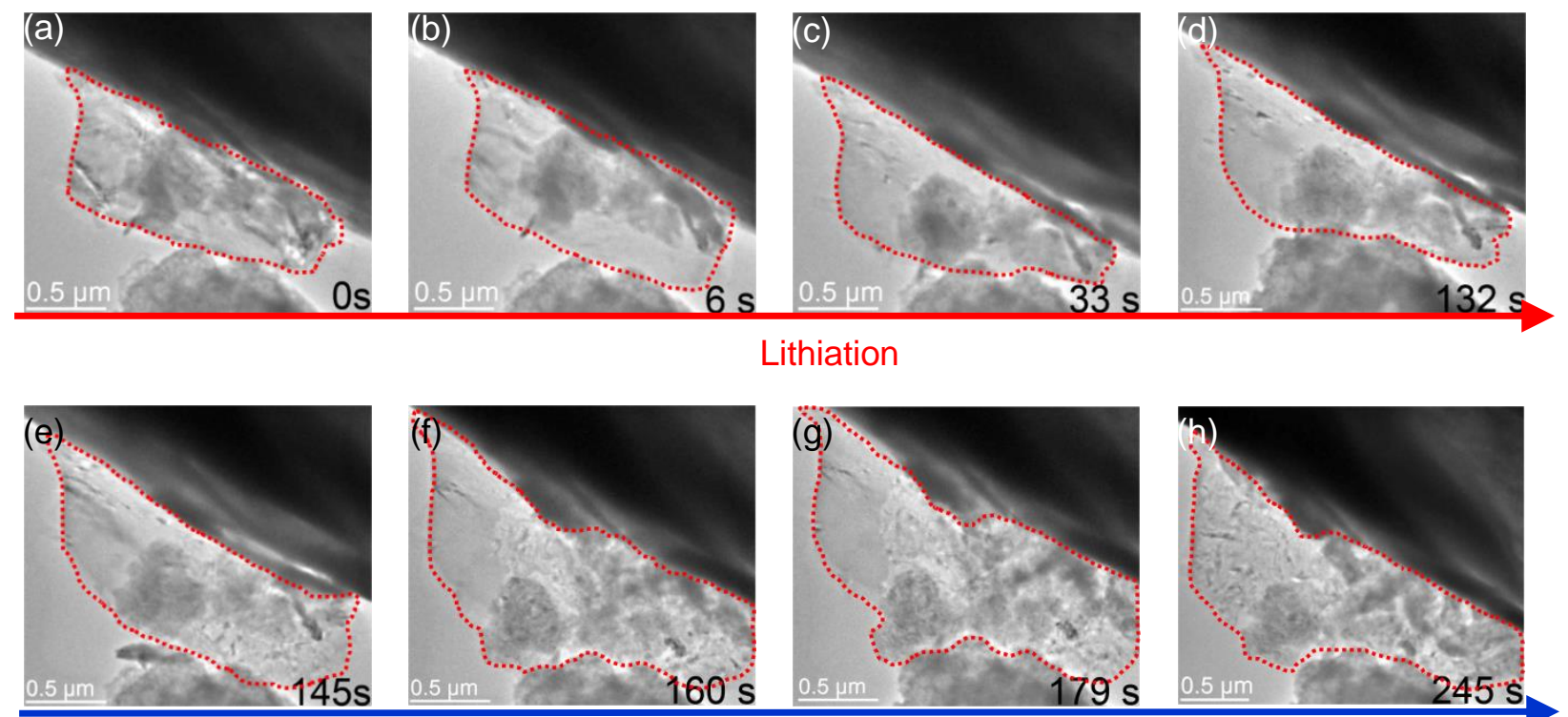

Delithiation

Figure S2. Time-resolved TEM images from video frames show the electrochemical processes of BP sheet during first lithiation/delithiation cycle. Pulverization and size increase can be easily observed during the delithiation process. 


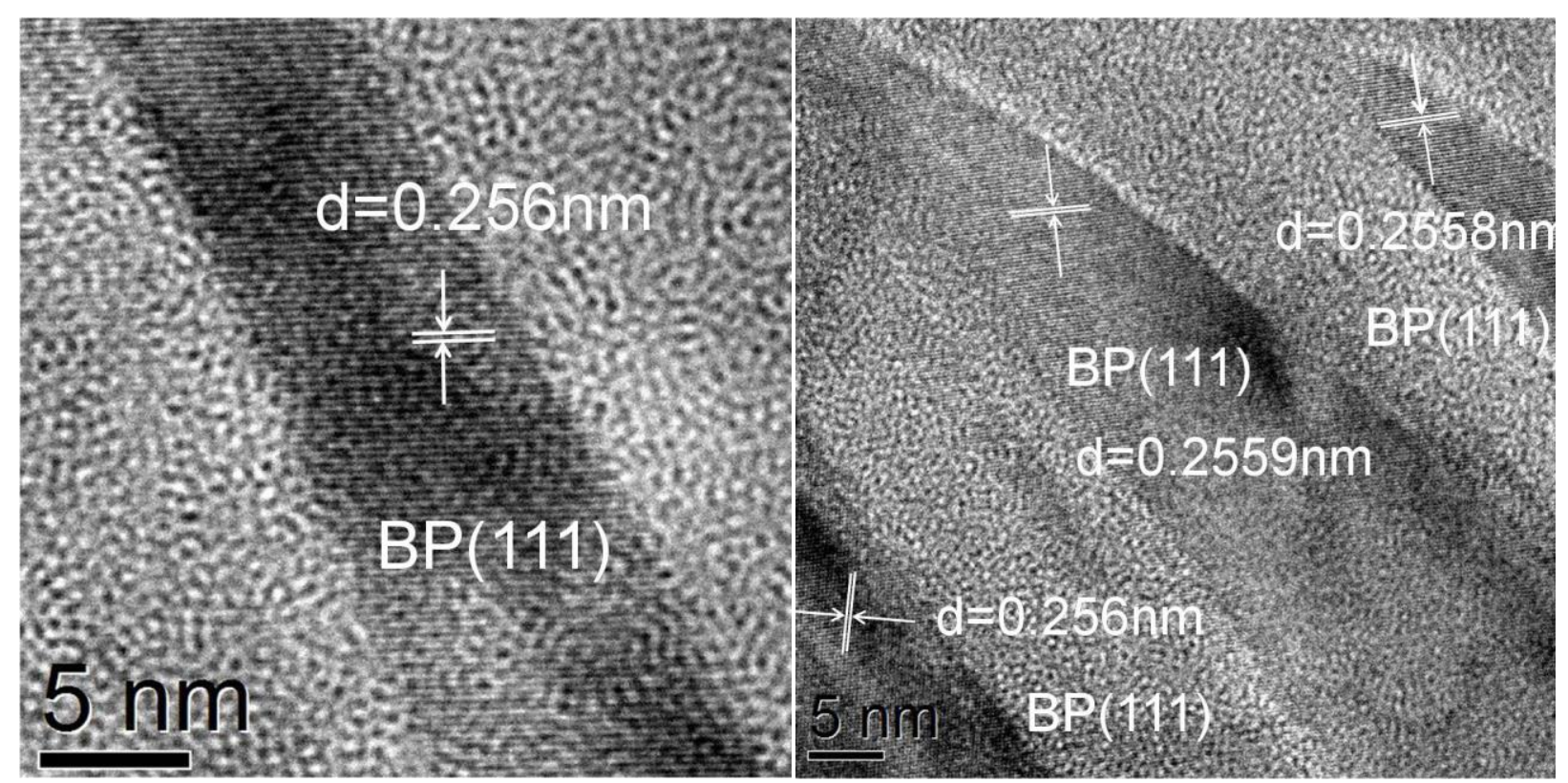

Figure S3. HRTEM images of BP anode after lithiation. 
Video V1 The dynamic process of BP sheet corresponding to Figure 2 during first lithiation.

Video V2 The dynamic process of BP sheet corresponding to Figure 4 during first lithiation.

Video V3 The dynamic processes of BP sheet corresponding to Figure 5 during first lithiation/delithiation cycle. 